\title{
Resultados de la aplicación de reciclado de pavimento asfáltico en los CIV de la localidad de Bosa y Suba. Estudio de caso
}

Fecha de recepción: 19 de octubre de 2018

Fecha de aprobación: 15 de diciembre de 2018

\author{
Adriana Patricia Parra \\ Corporación Universitaria Minuto de Dios - Uniminuto \\ aparra2@uniminuto.edu.co \\ Yury Yaquelin Ortega-Mora \\ Corporación Universitaria Minuto de Dios - Uniminuto \\ yortegamora@uniminuto.edu.co \\ Vladimir Acevedo-Pérez \\ Corporación Universitaria Minuto de Dios - Uniminuto \\ jhon.acevedo@uniminuto.edu
}

DOI: https://doi.org/10.21158/23823399.v6.n0.2018.2422

Cómo citar este artículo: Parra, A. P.; Ortega-Mora, Y. Y.; Acevedo-Pérez, V. (2018). Resultados de la aplicación de reciclado de pavimento asfáltico en los CIV de la localidad de Bosa y Suba. Estudio de caso. Revista Ontare, 6, (35-). DOI: https://doi.org/10.21158/23823399.v6.n0.2018.2422

\section{Resumen}

El deterioro de las vías es uno de los mayores inconvenientes que se presenta hoy en Bogotá. En los últimos años se ha evidenciado una problemática ambiental con los materiales asfálticos desechados. Una de las causas del desgaste de la carpeta de rodadura es el incremento del número de ejes equivalentes, ya que los pavimentos se

1 Ingeniera civil. Corporación Universitaria Minuto de Dios - Uniminuto. ORCID: https://orcid. org/0000-0002-5897-9050

2 Estudiante del programa de Ingeniería civil. Corporación Universitaria Minuto de Dios - uniminuto. ORCID: https://orcid.org/0000-0001-6191-949X

3 Ingeniero civil de la Universidad Católica de Colombia. Ingeniero topógrafo de la Universidad Distrital Francisco José de Caldas. Especialista en Diseño de Vías Urbanas, Tránsito y Transporte de la Universidad Distrital Francisco José de Caldas. ORCID: https://orcid.org/0000-0001-5337-3351 
diseñaron para un determinado número de vehículos, sin embargo, en la actualidad estos han aumentado, de manera que disminuyen la vida útil del material. Además del cambio climático — que es el agente que más influye en el deterioro de los pavimentos - también se cuenta con pérdida de biodiversidad, disminución del espesor de la capa de ozono y aumento de emisiones de $\mathrm{CO} 2$, lo que contribuye al deterioro ambiental. Por esta razón se investigan los diferentes métodos que existen a nivel mundial para realizar un reciclado de asfalto y la manera óptima de realizar su rehabilitación, después de analizar el comportamiento, la implementación, la factibilidad y los costos de todos los métodos.

De esta manera se pudo concluir que, en el caso de Bogotá, la implementación del RAP por medio de las entidades encargadas de la malla vial — tales como la Unidad de Mantenimiento Vial y el Instituto de Desarrollo Urbano - se realizó sin una revisión técnica, es decir, por medio de trabajos experimentales y con porcentajes altos de este material, lo que conllevó a que la carpeta asfáltica de los tramos intervenidos con este método presentara fatiga en un corto tiempo.

\section{Palabras clave}

Reciclaje; métodos de reciclaje; material asfáltico; reciclaje de pavimento; reciclaje de asfalto; sostenibilidad. 


\title{
Results of the implementation of asphalt pavement recycling in the Roadway Identification Codes of the localities of Bosa and Suba. Case study
}

\begin{abstract}
Nowadays, the deterioration of the roads is one of the biggest inconveniences in Bogotá. In recent years, studies have demonstrated an environmental problem that has to do with the asphalt materials that are discarded. One of the causes of the abrasion of the surface course is the increase in the number of equivalent axes, as pavements were designed for a certain number of vehicles, however, this number has now increased reducing the useful life of the material. In addition to climate change - which is the agent that most influences the deterioration of pavements there is also a loss of biodiversity, a decrease in the thickness of the ozone layer, and an increase in $\mathrm{CO} 2$ emissions, which also contributes to environmental deterioration. For this reason, the different methods for asphalt recycling that are available worldwide and the optimal way to perform its rehabilitation are investigated, after analyzing the behavior, implementation, feasibility, and costs of all the methods.

In this way, it was concluded that in the case of Bogotá, the implementation of the RAP through the entities in charge of the road network - such as the Road Maintenance Unit and the Urban Development Institute - was carried out without a technical review, that is, by means of experimental works and with high percentages of this material, entailing for the surface course of the sections that were intervened with this method to show abrasion in a short time.
\end{abstract}

\section{Keywords}

Recycling; recycling methods; asphalt material; pavement recycling; asphalt recycling; sustainability; Roadway Identification Codes (CIV by its abbreviation in Spanish). 


\title{
Resultados da aplicação de reciclagem de pavimentos asfálticos no CIV da cidade de Bosa e Suba. Estudo de caso
}

\begin{abstract}
Resumo
A deterioração das vías é um dos maiores inconvenientes que ocorrem hoje em Bogotá. Nos últimos anos, um problema ambiental foi evidenciado com os materiais asfálticos descartados. Uma das causas do desgaste da banda de rodagem é o aumento do número de eixos equivalentes, uma vez que os pavimentos foram projetados para um certo número de veículos, mas agora eles aumentaram, reduzindo a vida útil do material. Além das mudanças climáticas - que é o agente que mais influencia a deterioração dos pavimentos -, há também perda de biodiversidade, diminuição da espessura da camada de ozônio e aumento das emissões de CO2, o que contribui para a deterioração ambiental. Por esse motivo, investiga-se os diferentes métodos existentes a nível mundial para a reciclagem de asfalto e a maneira ideal de realizar sua reabilitação, após análise do comportamento, implementação, viabilidade e custos de todos os métodos.

Dessa forma, concluiu-se que, no caso de Bogotá, a implementação do RAP através das entidades responsáveis pela malha viária - como a Unidade de Manutenção Rodoviária e o Instituto de Desenvolvimento Urbano - foi realizada sem uma revisão técnica, ou seja, por meio de trabalhos experimentais e com altas porcentagens desse material, o que fez com que a malha asfáltica das seções intervenidas com esse método apresentasse desgaste em pouco tempo.
\end{abstract}

\section{Palavras-chave}

Reciclagem; métodos de reciclagem; material asfáltico; reciclagem de pavimentos; reciclagem de asfalto; sustentabilidade. 


\title{
Analyse des résultats de l'utilisation de goudron recyclé dans les CIV des municipalités de Bosa et Suba. Étude de cas
}

\begin{abstract}
Résumé
La détérioration des routes et des chaussées est l'une des principales problématiques affectant la mobilité de Bogotá. Un nouveau problème environnemental a de surcroît été constaté avec la mise au rebus de matériaux à base goudron. L'une des causes de l'usure prématurée du réseau routier urbain est l'augmentation du nombre de véhicules utilisant des voies conçues pour une quantité de déplacement déterminé, mais dont la croissance exponentielle réduit la durée de vie utile des matériaux. Outre le changement climatique, qui contribue largement à la détérioration des chaussées, on observe également une perte de biodiversité, une diminution de l'épaisseur de la couche d'ozone et une augmentation des émissions de $\mathrm{CO}_{2}$ qui contribuent à la dégradation de l'environnement. Pour cela, ce travail d'investigation étudie les différentes méthodes existantes au niveau international permettant le recyclage et la réutilisation du goudron et analyse le comportement, la mise en œuvre, la faisabilité et les coûts d'exécution des solutions existantes.
\end{abstract}

Dans le cas de Bogotá, il est ainsi possible d'affirmer que la mise en ouvre du PAR, réalisé par les acteurs publics en charge du réseau routier - l'Unité d'Entretien des Routes et l'Institut de Développement Urbain - a été faite sans examen technique préalable approfondis, ce qui laisse prévoir une détérioration accélérée des routes goudronnées de la capitale.

\section{Mots-clés}

Rrecyclage; méthodes de recyclage; asphalte; recyclage des chaussées; recyclage de l'asphalte; durabilité. 


\section{Introducción}

11 mundo enfrenta un problema común: los volúmenes de tráfico y las cargas por eje que aumentan día a día. Sin embargo, la inversión actual para el mantenimiento de las vías disminuye. La expectativa para realizar la rehabilitación se plantea en el desarrollo de métodos de bajo costo que no comprometan el medio ambiente. La presente crítica reúne los diferentes métodos utilizados en la actualidad en algunas partes del mundo para la recuperación vial. Con base en esto se opta por investigar los resultados de la metodología implementada en las localidades de Bosa y Suba, en Bogotá.

Los métodos utilizados en la actualidad son:

- Warm asphalt mixtures (WMA). De acuerdo con Ortega (2012):

Estas mezclas son diseñadas para colocarlas y compactarlas a una temperatura inferior a la convencional desde un rango de $100{ }^{\circ} \mathrm{C}$ y $140{ }^{\circ} \mathrm{C}$. Implementada en otros países como Francia, Alemania, Estados Unidos, Italia, China y otras naciones, los cuales muestran un interés importante en la producción de esta metodología, debido a los beneficios demostrados favoreciendo al medio ambiente.

- Mezclas modificadas con polímero

Rondón-Quintana et al. (2007) afirman que estas: Se adicionaron a la norma ASTM con la implementación de hule molido, aunque en la norma no se ha incluido otro tipo de polímeros reciclados como el polietileno expandido, el polietilentereftalato y otros, el producto residual de esos polímeros representa una mejora en la propiedad mecánica de la carpeta asfáltica, unas formas notables 
son: la resistencia dada a la deformación, la permeabilidad, la fricción neumático-pavimento y otras de menor relevancia. El asfalto de estas características tiene mejor capacidad de disipar la energía, por la organización generada con los polímeros.

- Residuos de construcción y demolición (RCD).

Estos se utilizan como agregados en el diseño de mezclas asfálticas en caliente, a fin de realizar un aprovechamiento de residuos de construcción y demolición que se utilicen a su vez como agregados en las mezclas asfálticas. En razón a lo anterior, es posible reemplazar de manera total los agregados naturales por artificiales usados en la elaboración de concretos asfálticos sin afectar sus parámetros mecánicos —esfuerzo-deformación-.

- Full depth reclamation (FDR).

La recuperación a profundidad es una técnica de rehabilitación de pavimento en la que la sección de pavimento flexible completo, y una parte predeterminada de los materiales subyacentes, se trituran, pulverizan o mezclan de manera uniforme, lo que da como resultado una capa de base estabilizada.

- Grano de caucho reciclado (GCR).

Según Diaz-Claros y Castro-Celis (2017):

El GCR es un material obtenido de las llantas en desuso de los vehículos automotores, que por lo general, tiene un destino no muy controlado ambientalmente en rellenos sanitarios, plantas térmicas y basureros a cielo abierto, entre otros, que generan un daño ambiental importante. Se obtiene mediante procesos de molienda de llantas usadas, disminuido en tamaño, este material es utilizado en diferentes obras de ingeniería civil, como lo 
son en rellenos de terraplenes, materiales de contención, pisos de parques, como modificador en las mezclas asfálticas, entre otros.

En las diferentes revisiones bibliográficas realizadas en esta investigación se encontró que el grano de caucho se ablanda y se expande a medida que va reaccionando con el asfalto. La adición de este a las mezclas produce un bitumen más espeso, lo cual tiene que ver con que se presente mayor resistencia al envejecimiento y a la oxidación. (p. 21)

Al analizar los métodos descritos para la recuperación de las vías que se encuentran afectadas por los volúmenes de tráfico y el cambio climático, este informe se enfoca en el método de reciclado de pavimentos asfálticos RAP, puesto que es el más viable en Bogotá, dadas las condiciones climáticas, ambientales y económicas.

Al respecto, Reyes-Ortiz, Berardinelli, Álvarez, Carvajal-Muñoz y Fuentes señalan (2012):

RAP es un material introducido en la rehabilitación de carreteras que consiste en la reutilización de materiales procedentes de las capas que conforman la estructura de un pavimento que ha estado en servicio y ha perdido algunas de sus capacidades iníciales por el efecto de la temperatura, el efecto del medio ambiente y el impacto de las cargas vehiculares que ha soportado, pero que pueden mejorarse al tratarse o estabilizarse con materiales vírgenes para conformar nuevas capas. (p. 379)

Se entiende como «reciclado de pavimentos» la reutilización de los materiales procedentes de la demolición de las capas de pavimentos que ya han estado en servicio y han disminuido sus capacidades. Este material reciclado se utiliza en la construcción de nuevas vías. 
El reciclado de pavimentos asfálticos se realiza sobre materiales deteriorados que han perdido, en gran medida, sus propiedades iniciales. El reciclado es, en unos casos, una alternativa al fresado y la reposición de carpetas de rodadura o a la construcción; en otros constituye un aprovechamiento de materiales fresados para darle otros usos como complemento de la base o la subbase (Cedex, 2011).

El hecho de que una gran parte del sistema vial de Bogotá y otras capitales departamentales se encuentre en mal estado, es la razón por la que lo ubicamos como un campo útil para su reciclaje al rescatar la superficie de pavimento y, luego de mezclarlo, pasarlo a moliendas y nuevas mezclas, de modo que se le den los tratamientos físico-calóricos que permitan mejores materiales en una nueva aplicación. Esto puede también ser útil en la recuperación de taludes o como relleno en esa nueva capa.

En los países de la Unión Europea se ha logrado una restitución de las capas de rodadura y sustento que supera el $15 \%$ en promedio. Por su parte, en el Cono Sur de América se encuentran sustituciones mayores al $27 \%$, y en América del Norte se ha llegado a una cifra del $80 \%$ de restitución.

En el territorio colombiano se han re realizado otros proyectos con RAP, como, por ejemplo:

La vía Puerto López-Puerto Gaitán (Meta), Quibdó-Yuto (Chocó), Medellín-Santuario (Antioquia), Cali-Loboguerrero (Valle del Cauca), Manizales-Mesones (Caldas), Autopista Bogotá-Villavicencio (Cundinamarca). En Bogotá se han llevado a cabo algunos trabajos de reciclaje en frío en vías principales, en el departamento de Antioquia también se ha llevado a cabo en la vía al mar destacándose los tramos: Uramita-Dabeiba-Mutatá-Chigorodó y El Tres-Turbo. (Sánchez, 2012) 
Al estudiar diferentes informes sobre el tema hemos encontrado que es necesario descartar los materiales de mayor edad de uso, por lo cual el análisis más interesante lo localizamos en lo extraído del tramo de la vía Buga-Tuluá, cuya rehabilitación utilizó un 40 \% de RAP.

Este sistema ha encontrado el respaldo del Instituto de Desarrollo Urbano de Bogotá (IDU), en el que lo radican dentro de los procesos recomendados para la recuperación de vías con la utilización de medios mecánicos de molienda que generan características interesantes de soporte a las cargas pesadas y mejor resistencia a la humedad. Así mismo, existen ahorros significativos al comparar el material reciclado con el uso de materiales vírgenes que pueden representar entre un $20 \%$ y un $40 \%$ de menor inversión.

La metodología del RAP inicio en Bogotá bajo el convenio 1292:

El convenio Interadministrativo de cooperación $\mathrm{N}^{\circ} 1292$, tiene por objeto la ejecución directa por parte de las alcaldías locales y demás entidades que son parte del mismo, de la construcción, reconstrucción, rehabilitación y mantenimiento de las vías locales, espacio público, adecuación de andenes para la movilidad de las personas en situación de discapacidad y la arborización de las zonas intervenidas, en desarrollo de la línea de inversión localmalla vial; con cargo al presupuesto de los fondos de desarrollo local, conforme a las decisiones de los cabildos ciudadanos sobre priorización de segmentos viales a intervenir. (Veeduría Distrital, 2 de diciembre de 2015, p.1)

A nivel nacional, la normatividad concerniente al reciclaje de pavimentos asfálticos se enmarca dentro de las especificaciones del IDU ET-2005, en sus secciones 450-05 y 454-0, además de las especificaciones técnicas del Instituto Nacional de Vías (Invias), en su artículo 461-07 
y 462-07, dado que son estas últimas las de mayor importancia y las más utilizadas a nivel nacional (Restrepo y Stephens, 2015).

\section{Técnicas de reciclado RAP}

\subsection{Reciclado en planta caliente}

Se refiere únicamente a mezclas bituminosas. Para el reciclado en caliente las mezclas se retiran de la vía y se transportan a un depósito de fabricación en el que se clasifican y se mezclan en caliente con árido virgen y betún, de modo que se obtiene la nueva mezcla compuesta por al menos un $40 \%$ de material reciclado. A fin de procesar el material recuperado se puede realizar en la planta con el objeto de mejorar sus características y obtener un mayor porcentaje de reciclado. Por lo general, en planta se trabajan tres fases: triturado, clasificación y homogeneización.

Durante el triturado se procesan bloques enteros de pavimento, así como grandes terrones procedentes de fresado con el objetivo de conseguir tamaños menores. Esto se puede llevar a cabo tanto con la utilización de unidades móviles ubicadas en obra como con maquinaria instalada en la planta. Si el material permanece acopiado un largo periodo de tiempo, es posible que sea necesario volver a triturarlo, dado que se puede apelmazar, especialmente si las temperaturas son altas y la exposición solar prolongada. Para mejorar la homogeneidad de las diferentes fracciones tras el fresado o machaqueo, se recomienda la clasificación del material de acuerdo con los tamaños exigidos — cribado-

El almacenamiento en acopios separados exige una gran disponibilidad de espacio en la planta. Los acopios pueden clasificarse de acuerdo con sus diferentes características: tamaño máximo de partícula, granulometría, tipo de betún, origen del material, etc. Dado que el contenido de 
agua puede reducir el porcentaje de utilización del material, se aconseja que los acopios se encuentren techados (Cedex, diciembre de 2011).

\subsection{Reciclado en planta en frío}

No presenta características especiales. Se lleva a cabo con áridos procedentes de capas granulares o con mezclas fresadas de las capas del pavimento. El uso de este tipo de técnicas puede llegar a permitir una tasa de reciclaje del $100 \%$.

Cuando se reutiliza para capas bituminosas, el material se mezcla con emulsión en plantas continuas de mezcla en frío, o bien en plantas de grava-cemento modificadas para incorporar la emulsión. El proceso de fabricación es análogo al de una grava-emulsión. Las únicas limitaciones en el proceso son las que puedan deducirse de la granulometría o de los excesos de pegante en el material reciclado, las cuales pueden exigir una corrección por aportación de material granular. El ahorro conseguido depende de nuevo de las condiciones de la operación.

Esta técnica se puede utilizar para la construcción de capas de base, aunque también para capas intermedias, e incluso de rodadura en carreteras secundarias. El espesor de las capas es variable y se puede adaptar a los requisitos técnicos. Si el espesor de la capa supera los $20 \mathrm{~cm}$, se recomienda dividir su puesta en obra en dos capas más delgadas. Cuando se ha completado la producción en planta, la mezcla final se transporta y se pone en obra con maquinaria convencional. Para la compactación se pueden utilizar rodillos vibratorios pesados.

Una desventaja de este tipo de técnicas cuando se utiliza emulsión bituminosa o cemento es el tiempo de curado, el cual depende de las condiciones de temperatura y humedad, que, a su vez, pueden variar desde días a meses. 


\subsection{Reciclado in situ en caliente}

Es una técnica aplicable a materiales bituminosos. El reciclado se realiza in situ con unos equipos especiales, provistos de calefactores, que elevan la temperatura de la superficie del pavimento y facilitan la abrasión del material. El elemento básico del equipo es el escarificador o fresador-calentador. Por lo general, el calentador consiste en unos paneles de rayos infrarrojos que calientan la superficie a temperaturas comprendidas entre $120^{\circ} \mathrm{C}$ y $160^{\circ} \mathrm{C}$. Suele haber una serie de paneles que precalientan la superficie y eliminan la humedad, y una o dos series - si se reciclan más de 2-3 cm, ya que suele hacerse en dos etapas- que aplican la temperatura final. El equipo de escarificado está provisto de dientes de acero con puntas de carborundo, aunque a veces se utilizan fresadoras convencionales. El material suelto se mezcla con un betún blando o un agente rejuvenecedor. La técnica puede llevarse a cabo con o sin aportación de árido nuevo, de modo que realiza la operación de mezcla el mismo equipo. La extensión debe llevarse a cabo con una máquina convencional que acompaña al equipo. Por su parte, la compactación debe realizarse de forma muy rápida, y es un punto crítico de la operación.

La ventaja de este método frente al reciclado en frío es que degrada menos los áridos, y que aprovecha más la capacidad aglomerante del betún de la mezcla antigua.

Los principales inconvenientes son que no soluciona problemas estructurales y que el calentamiento oxida y fragiliza el betún. La emisión de humos, los peligros de quemaduras a los operarios, o los sobrecalentamientos por paradas de la máquina han limitado también en parte la aplicación (Cedex, diciembre de 2011). 
Figura 1. Rangos de temperatura para producción de mezclas asfálticas

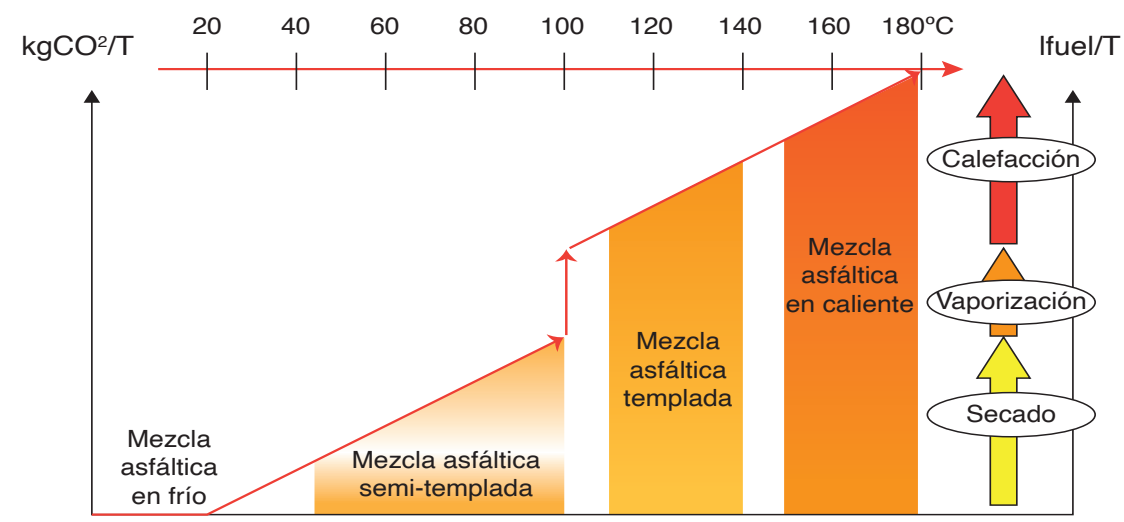

Fuente. Porot, 2008.

\subsection{El reciclado en frío in situ}

Se realiza con equipos específicos multifunción que fresan el material y lo mezclan con agua y los conglomerantes o ligantes seleccionados, de modo que se realiza todo el proceso a temperatura ambiente. Con la técnica del reciclado in situ se pueden reciclar capas de mezcla bituminosa, de materiales granulares o tratados con cemento, o conjuntos de estas capas. Es muy importante tener en cuenta que solo conviene reciclar materiales deteriorados y no zonas sanas que, por lo general, tendrán unas características mecánicas superiores a las del material reciclado. Con esta técnica se pueden alcanzar porcentajes del $100 \%$ de reciclaje, si bien es cierto que es habitual la adición de una capa bituminosa superficial.

Las ventajas principales frente al reciclado en caliente son: la falta de emisión de humos, el empleo de menos energía y la posibilidad de reciclar un mayor espesor de firme.

Los inconvenientes son la falta de aprovechamiento de la capacidad aglomerante del betún antiguo, el tiempo necesario para la apertura al trá- 
fico y la regularidad final de la capa, ya que al afectar, en general, a mayores espesores con relación a otros tipos de reciclados, haberse degradado más la granulometría y realizarse en frío, suele llevar a compactaciones difíciles (Cedex, diciembre de 2011).

\section{Desarrollo de la experiencia}

L

a metodología para el sistema RAP consiste en la recuperación y el vial, a causa de los de mezcla y aplicación. La aplicación de esta técnica, en lo que comprende del 2005 en adelante, según lo reglamenta la Resolución 1959 del 18 de mayo del 2006, se titula o describe como «el reciclado de pavimentos asfálticos con el tratamiento o estabilización con emulsión asfáltica, asfalto espumado y cemento portland».

Figura 2. Serviciabilidad del pavimento-comportamiento

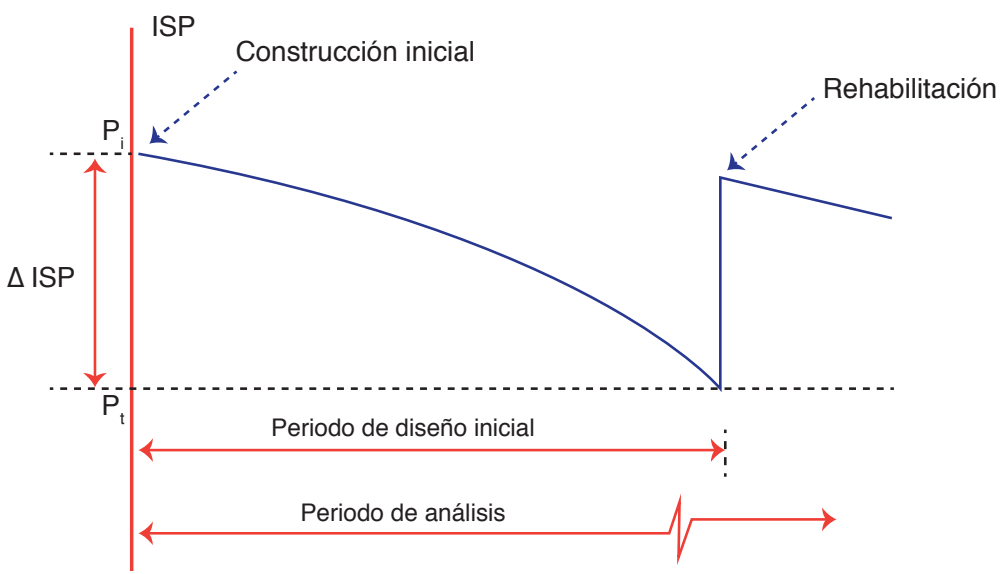

Fuente. Sánchez, s. f. 


\subsection{Ciclo de vida}

Es un proceso que permite evaluar las cargas ambientales asociadas a un producto, un proceso o una actividad al identificar y cuantificar el uso de materia y energía, así como los residuos que genera. En este proceso se realizan diversos estudios energéticos en los que se valora la eficiencia de determinadas fuentes de energía, luego se incorporan nuevos conceptos como, por ejemplo, el consumo de recursos naturales, las emisiones atmosféricas, las emisiones al agua o la generación de residuos.

Los métodos de Análisis de Ciclo de Vida pretenden analizar el impacto que ocasionan en cada una de las fases de su vida.

Lo fundamental es cuantificar en magnitudes comparativas dicho impacto - por ejemplo, las emisiones de gases invernadero se traducen en cantidades equivalentes de $\mathrm{CO}_{2}-$.

Figura 3. Ciclo de vida de una calzada

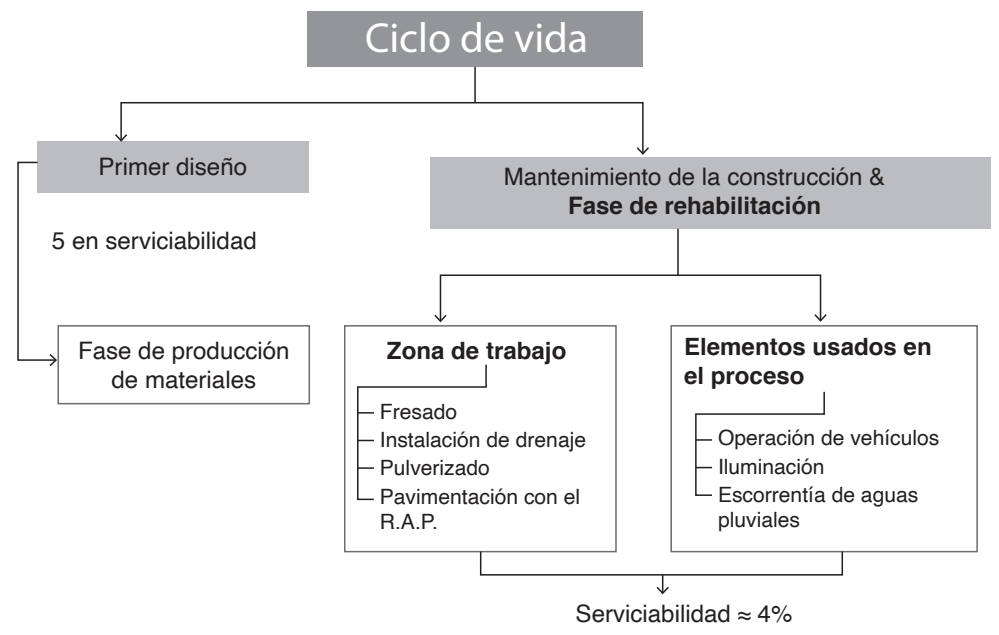

Fuente. Elaboración propia. 
En general, tanto la institucionalidad — cuando sus labores afectan el plan vial nacional - como los centros de investigación universitaria y de ingeniería civil han tratado el tema en profundidad en la búsqueda de la recuperación de la mayor cantidad de material del sistema vial nacional, estropeado pero existente; esto significa economías importantes al evitar gran parte de los escombros que atentan contra el medio ambiente.

Después de revisar diferentes fuentes sobre el desarrollo del tema y tener en cuenta las especificaciones técnicas del IDU, se realizaron unas visitas a los funcionarios de esta entidad pública con el fin de solicitar información reciente de los tramos intervenidos y sus respectivas fórmulas de trabajo. Con la información que se obtuvo se verifica que el método del RAP es una alternativa eficiente de recuperación en las vías de Bogotá con bajos volúmenes de tránsito. De igual forma, se observa que la implementación del método en una etapa anterior no cumplió con las especificaciones técnicas, lo cual llevó a un deterioro prematuro de las calzadas intervenidas.

En la actualidad, las instituciones competentes trabajan en la búsqueda y el desarrollo de una fórmula de trabajo estandarizada que brinde las condiciones óptimas en el momento de intervenir cualquier eje vial de la ciudad.

Nosotros, como comunidad académica investigativa, realizamos un seguimiento a las calzadas intervenidas recientemente con este método. Ahora bien, dependiendo del comportamiento a través del tiempo se realizará un estudio de las patologías presentadas con el fin de comprobar la efectividad de la aplicación de la fórmula de trabajo.

Al realizar la revisión de vías en Bogotá intervenidas con la técnica del RAP fue posible evidenciar que la práctica, inicialmente, se realizó con conocimientos empíricos. Luego de una investigación más profunda, las entidades públicas interesadas en el tema, como, por ejemplo, la Uni- 
dad de Mantenimiento Vial (UMV), se dio a la tarea de trabajar con instituciones académicas en el propósito de buscar el mejor diseño de la mezcla, o lo que hemos denominado «la fórmula de trabajo», con el objetivo de buscar la cantidad exacta de material reciclado que se debe agregar al nuevo diseño, y así este brinde las características necesarias como si fuera material virgen.

La UMV realiza actualmente tramos de prueba en algunas vías de Bogotá, tal como se refiere en la tabla 1.

Tabla 1. Tramo intervenido por rehabilitación en el 2014

\begin{tabular}{|c|c|c|c|c|c|}
\hline CIV & $\begin{array}{c}\text { Nombre } \\
\text { UPZ }\end{array}$ & Tramo & Desde & Hasta & $\begin{array}{l}\text { Longitud de } \\
\text { CIV }\end{array}$ \\
\hline 7002077 & $\begin{array}{c}\text { Bosa } \\
\text { Occidental }\end{array}$ & CL 69A s & KR $88 C$ & KR 88A & $37 \mathrm{~m}$ \\
\hline 7002132 & $\begin{array}{c}\text { Bosa } \\
\text { Occidental }\end{array}$ & CL 69A s & KR 88A & KR $88 \mathrm{Bis}$ & $19 \mathrm{~m}$ \\
\hline 7002166 & $\begin{array}{c}\text { Bosa } \\
\text { Occidental }\end{array}$ & CL 69As & KR $88 \mathrm{Bis}$ & KR 88 & $12 \mathrm{~m}$ \\
\hline 7002249 & $\begin{array}{c}\text { Bosa } \\
\text { Occidental }\end{array}$ & CL 69A s & KR 88 & KR 88Q & $22 \mathrm{~m}$ \\
\hline 30000604 & $\begin{array}{c}\text { Bosa } \\
\text { Occidental }\end{array}$ & CL 69As & KR $88 Q$ & KR 88P & $11 \mathrm{~m}$ \\
\hline 7002282 & $\begin{array}{c}\text { Bosa } \\
\text { Occidental }\end{array}$ & CL 69As & KR 88P & KR $88 N$ & $21 \mathrm{~m}$ \\
\hline 7002345 & $\begin{array}{c}\text { Bosa } \\
\text { Occidental }\end{array}$ & CL 69A s & KR $88 \mathrm{~N}$ & KR 88L & $18 \mathrm{~m}$ \\
\hline 30000606 & $\begin{array}{c}\text { Bosa } \\
\text { Occidental }\end{array}$ & CL 69A s & KR $88 \mathrm{~L}$ & $\mathrm{KR} 88 \mathrm{~K}$ Bis & $15 \mathrm{~m}$ \\
\hline 30000607 & $\begin{array}{c}\text { Bosa } \\
\text { Occidental }\end{array}$ & $\begin{array}{c}\text { KR } 87 \mathrm{~K} \\
\text { Bis }\end{array}$ & CL $70 \mathrm{~S}$ & CL 69A s & $128 \mathrm{~m}$ \\
\hline 30000607 & $\begin{array}{c}\text { Bosa } \\
\text { Occidental }\end{array}$ & $\begin{array}{c}\text { KR } 87 \mathrm{~K} \\
\text { Bis }\end{array}$ & CL $70 \mathrm{~S}$ & CL 69A s & $128 \mathrm{~m}$ \\
\hline
\end{tabular}


Fuente: Alcaldía Local de Bosa, 2018.

Al realizar la inspección visual, después de cinco años de intervención, solo se encontró un hundimiento de severidad media que comprometía menos del $1 \%$ del CIV 7002077, por lo cual se afirma que es importante la fórmula de trabajo para el uso de esta técnica en tramos de volúmenes de tránsito mayores.

Tabla 2. Tramo intervenido por rehabilitación en el 2018

\begin{tabular}{|c|c|c|c|c|c|}
\hline CIV & $\begin{array}{c}\text { Nombre } \\
\text { UPZ }\end{array}$ & Tramo & Desde & Hasta & $\begin{array}{c}\text { Longitud } \\
\text { de CIV }\end{array}$ \\
\hline 7007659 & $\begin{array}{c}\text { Bosa } \\
\text { Central }\end{array}$ & KR 77J & CL 65G s & CL 65G Bis A s & $47 \mathrm{~m}$ \\
\hline 7007670 & $\begin{array}{c}\text { Bosa } \\
\text { Central }\end{array}$ & KR 77J & $\begin{array}{c}\text { CL 65G Bis } \\
\text { A s }\end{array}$ & CL 65H s & $47 \mathrm{~m}$ \\
\hline 7007684 & $\begin{array}{c}\text { Bosa } \\
\text { Central }\end{array}$ & KR 77J & CL 65H s & CL 65I s & $45 \mathrm{~m}$ \\
\hline 7007699 & $\begin{array}{c}\text { Bosa } \\
\text { Central }\end{array}$ & KR 77J & CL 65I s & CL 65J s & $108 \mathrm{~m}$ \\
\hline 7007720 & $\begin{array}{c}\text { Bosa } \\
\text { Central }\end{array}$ & KR 77J & CL 65J s & & $144 \mathrm{~m}$ \\
\hline 7007731 & $\begin{array}{c}\text { Bosa } \\
\text { Central }\end{array}$ & KR 77J & CL 68 s & CL 69A s & $133 \mathrm{~m}$ \\
\hline
\end{tabular}

Fuente. Corredor, 2018.

Si se tiene en cuenta que esta vía se rehabilitó y entregó a principios del 2018, se realizó una inspeccion visual en abril del 2019 en la que se encontró un daño menor al $2 \%$ en todo el tramo. Esto quiere decir que el uso de asfalto reciclado es una opción favorable, pero se insiste en que se debe implementar con fórmula de trabajo, puesto que a simple vista se considera exitoso el tramo de prueba, pero en esto también influye el flujo 
de tránsito, por lo que es recomendable no realizar este tipo de intervenciones en vías principales, ya que las patologías se pueden presentar en menor tiempo.

Figura 4. Estado de las vías intervenida con RAP, Suba-Lisboa calle 134 \# 151A

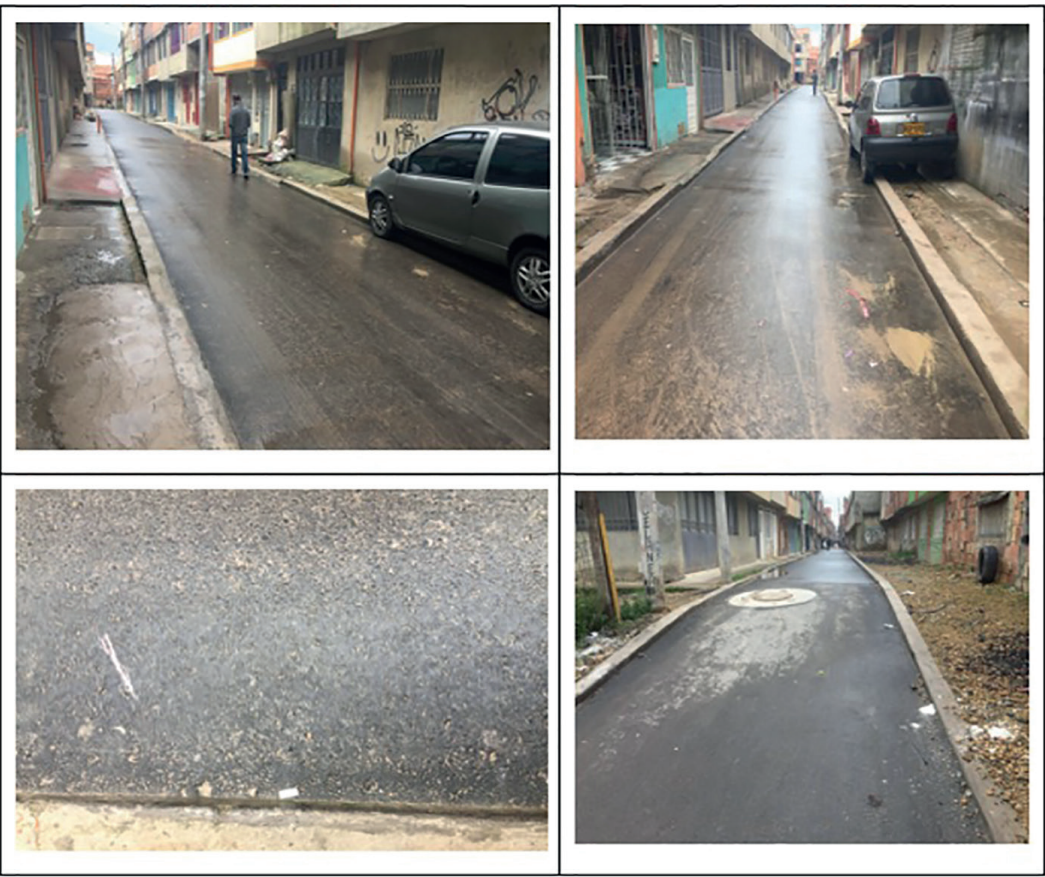

Fuente. Elaboración propia.

En la localidad de Suba, barrio Lisboa, en la calle 134 entre carreras 151A y 152, se llevó a cabo la implementación de la técnica del RAP, la cual, afirma la UMV, se realizó de manera correcta (CIV 11003861). La figura 4 presenta imágenes de los trabajos adelantados allí, en esta dirección. 
Con base en lo encontrado en los CIV de la localidad de Bosa y Suba se presentan unas ventajas y unas limitantes.

Algunas ventajas son:

- Bajos costos. Con estas técnicas se consigue un ahorro de los materiales naturales, lo que conlleva una ventaja tanto económica como medioambiental.

- Reduce el impacto ambiental. Las mezclas en las que se utiliza el RAP reducen de forma notable la emisión de $\mathrm{CO} 2$ en el proceso de producción e implementación.

- Reducción o eliminación de gases y olores. El reciclado de los materiales constitutivos de los pavimentos lleva consigo la conservación de los recursos naturales y el ahorro de espacio en vertederos públicos. Al tratar de reutilizar los materiales del pavimento es necesario analizar la posible presencia en su composición de elementos potencialmente peligrosos para el medio ambiente.

- Disponibilidad del material. A diario se realiza el fresado de diferentes capas de rodadura y estos materiales se trasladan a lugares de depósito o desechados en lugares de uso público.

- Los materiales con los que se realiza la rehabilitación. Contienen un porcentaje de reciclado, no contraminan, consumen poca energía en su ciclo de vida, son duraderos y económicos.

- Facilidad constructiva

Algunas limitantes son:

- Mal manejo del material. El material se remueve de la vía y se traslada a los lugares de depósito. Allí se mezcla con diferente material de diferentes tiempos de retirado y uso. 
- Fórmula de trabajo. Aún no existe registro acerca de la cantidad adecuada de material que se debe agregar al nuevo diseño, no obstante, al trabajar con un $40 \%$ de material reciclado la vía presenta características optimas de serviciabilidad.

\section{Conclusiones}

$\mathrm{E}$ $\mathrm{n}$ el momento de la implementación del RAP, por primera vez en Bogotá, por medio de las entidades encargadas de la malla vial tales como la Unidad de Mantenimiento Vial y el Instituto de Desarrollo Urbano_- esta se realizó sin una revisión técnica, es decir, por medio de trabajos experimentales y con porcentajes altos de este material; por ende, la carpeta asfáltica de los tramos intervenidos con este método presentó fatiga en un corto tiempo.

Según diferentes investigaciones realizadas, la utilización adecuada del RAP no solo es amigable con la naturaleza, sino que su principal atractivo es que reducen de forma significativa los gastos en la construcción.

En algunos países la capa asfáltica envejecida se considera un desperdicio $\mathrm{y}$, por ende, se desecha, puesto que ya ha cumplido su vida útil. Esto genera impactos negativos en el medio ambiente, razón por la cual se plantea la investigación de los estudios sobre el uso del RAP en Colombia $\mathrm{y}$, de esta forma, proporcionar un indicio de cómo formular la ecuación de trabajo para Bogotá.

Como respuesta a la rehabilitación del tramo en Bosa Central, se incrementó el paso vehicular, dado que antes de la intervención se buscaban vías alternas en razón a su deterioro. 
El uso de asfalto reciclado en un porcentaje menor al $30 \%$ arrojó un resultado relativamente positivo. De igual forma, se debe trabajar con una fórmula de trabajo para mejorar los efectos y estar en capacidad de implementar el uso en vías con volumen de tránsito mayor.

\section{Referencias}

Alcaldía Local de Bosa. (2018). Vías intervenidas con la técnica del RAP en Bogotá D.C, localidad de Bosa. Alcaldía Local de Bosa.

Cedex. (Diciembre de 2011). Reciclado de pavimentos asfalticos RAP. Ficha técnica. Recuperado de http://www.cedex.es/NR/ rdonlyres/26C518BE-1802-4803-82B1-FBE634B03B03/119932/RECICLADODEPAVIMENTOSASFALTICOS.pdf20de\%202012.pdf

Corredor, J. (2018). CIV del tramo las Torres en la UPZ Bosa Central. Alcaldía Local de Bosa.

Diaz-Claros, C. M.; Castro-Celis, L. C. (2017). Implementación del grano de caucho reciclado ( $\mathrm{gcr}$ ) proveniente de llantas usadas para mejorar las mezclas asfálticas y garantizar pavimentos sostenibles en Bogotá (Tesis de pregrado). Universidad Santo Tomás. Bogotá, Colombia.

Ortega, O. (2012). Propuesta para la implementación de mezclas asfálticas tibias en la ciudad de Medellín. (Tesis de especialización) Universidad de Medellín. Colombia, Recuperado de http://repository.udem. edu.co/

Porot, L. (2008). Mezclas asfálticas a más bajas temperaturas. Ponenecia presentada en $X X X V$ Reunión del Asfalto. Rosario, Argentina. Comisión Permanente del Asfalto.

Resolución 1959 de 2006. (18 de mayo de 2006). Por la cual se adopta el manual especificaciones técnicas generales de materiales y construc- 
ción para proyectos en infraestructura vial y de espacio público en Bogota, D.C., IDU ET 2005. Alcaldía Mayor de Bogotá.

Restrepo, H. A.; Stephens, S. A. (2015). Estudio de las ventajas económicas del reciclaje frío in situ de pavimentos asfalticos (tesis de especialización). Especialización en Vías y Transporte, Universidad de Medellín. Colombia.

Rondón-Quintana, H. A.; Reyes-Lizcano, F. A.; Figueroa-Infante, A. S.; Rodríguez-Rincón, E.; Real-Triana, C. M.; Montealegre-Elizalde, T. A. (2012). Estado del conocimiento del estudio sobre mezclas asfálticas modificadas en Colombia. Infraestructura Vial, 10(19), 10-20. DOI: https://doi.org/10.1016/j.sbspro.2012.09.889

Reyes-Ortiz, O.; Berardinelli, E.; Álvarez, A. E.; Carvajal-Muñoz, J. S.; Fuentes, L. G. (2012). Evaluation of hot mix asphalt mixtures with replacement of aggregates by reclaimed asphalt pavement (RAP) material. Procedia-Social and Behavioral Sciences, 53, 379-388. DOI: https://doi.org/10.1016/j.sbspro.2012.09.889

Sánchez, J. (s. f.). Construcción y rehabilitación de vías urbanas en Colombia pavimento asfáltico flexible (RAP). Recuperado de https://bit. ly/2WYL6Py

Veeduría Distrital. (2015). Informe de seguimiento al convenio interadministrativo de cooperación n.o 1292 de 2012 y revisión de los contratos interadministrativos de interventoría nos. 382 y 387 de 2013. Recuperado de https://bit.ly/2UQr6Mn 